\title{
Convergence of Mechanosensory Afferents from Different Classes of Exteroceptors onto Spiking Local Interneurons in the Locust
}

\author{
M. Burrows and P. L. Newland \\ Department of Zoology, University of Cambridge, Cambridge CB2 3EJ, United Kingdom
}

Tactile stimuli to a leg of a locust are detected by two classes of exteroceptors: trichoid sensilla (tactile hairs), which are purely mechanoreceptors, and basiconic sensilla, which serve a dual role as mechano- and chemoreceptors. The trichoid sensilla have the longer shafts, but because the two types of receptors are intermingled over the surface of a leg, both can be excited when the leg contacts an obstacle. This article analyzes the mechanisms by which the tactile sensory information from these two classes of receptor is collated in the CNS so that spatial information is preserved.

The mechanosensory afferents from both types of receptors on a hind leg make convergent connections that are excitatory and apparently direct with the same spiking local interneurons in a midline population of the metathoracic ganglion. The anatomy of the afferent projections suggests that the synapses from the two types of receptor are made onto the same region of branches of an interneuron. The slower conduction velocity of the spikes of the basiconic afferents compared to that of the trichoid afferents means that a mechanical stimulus to the tibia will first produce a depolarization caused by the trichoid afferents, followed up to $20 \mathrm{msec}$ later by a depolarization caused by the basiconic sensilla.

Each interneuron is excited by a contiguous and overlapping array of trichoid and basiconic receptors that form its receptive field. Different interneurons have different receptive fields such that the surface of the leg is mapped as a series of overlapping receptive fields. Within a receptive field the contribution of individual receptors can be markedly different: some basiconic receptors evoke large-amplitude T.PSPs so that each afferent spike can cause a spike in the interneuron, whereas others generate small EPSPs that must sum with other inputs to evoke a spike. The amplitudes of the EPSPS generated by different receptors within a receptive field form gradients that are distributed according to the axes of the leg. The most effective basiconic and the most effective trichoid sensilla within a particular receptive field can be at the same or at different locations on the leg.

Tactile sensory information from different types of receptor on one region of a leg is therefore collated initially by

Received July 30, 1993; accepted Oct. 14, 1993.

This work was supported by Grant NS 16058 from NIH, by the Wellcome Trust, and by an equipment grant from the Royal Society to P.L.N. We thank our Cambridge colleagues and Dr. D. O'Dell for their helpful comments on the manuscript.

Correspondence should be addressed to Professor M. Burrows, Department of Zoology, University of Cambridge, Downing Street, Cambridge CB2 3EJ, England. Copyright (c) 1994 Society for Neuroscience $0270-6474 / 94 / 143341-10 \$ 05.00 / 0$ particular spiking local interneurons. This organization ensures summation between the inputs from the different types of receptors and should enhance sensitivity. It also preserves spatial information for use in tactile reflexes, and could ensure that appropriately directed movements are made in response to the parallel information from the chemosensory afferents that innervate the basiconic sensilla.

[Key words: sensory convergence, mechanoreceptors, local reflexes, insect, grasshopper, receptive field]

Tactile signals from the surface of a limb are important in providing information about external impediments during locomotion, and in signaling that a limb should be moved away from a potentially harmful stimulus. The limbs of different animals are thercfore equipped with a variety of exteroceptors, the sensory neurons of which respond to different features of a mechanical stimulus, and influence the expression of the motor output controlling the movements of these limbs. Mammalian skin contains several different types of receptor that contribute to the sensation of touch, the afferents of which are mapped in the CNS according to receptor type and location. For example, hair follicle afferents are mapped in a somatotopic fashion in the spinal cord (Koerber and Brown, 1982; Nyberg and Blomqvist, 1985; Shortland et al., 1989; Brown et al., 1991), whereas the terminals of high- and low-threshold $\delta$ afferents project in different laminae (Light and Perl, 1979). A similar somatotopic mapping of tactile afferents also occurs in insects (Murphey et al., 1980; Pflüger et al., 1981; Johnson and Murphey, 1985; Levine et al., 1985; Kent and Levine, 1988; Peterson and Weeks, 1988; Newland, 1991a) with a segregation of tactile and proprioceptive afferents in different regions of neuropil (Pflüger et al., 1981; Burrows, 1987; Murphey et al., 1989; Merritt and Murphey, 1992; Burrows and Newland, 1993).

The neural circuitry processing the signals from these tactile receptors must preserve spatial information, because different movements can result from stimulation of receptors on different regions of a leg (Siegler and Burrows, 1986). In both vertebrates and arthropods, the afferents from the exteroceptors generally do not make monosynaptic connections with the motor neurons but instead exert their effects through networks of interneurons (Siegler and Burrows, 1983; Brown et al., 1987; Burrows, 1992; Nagayama and Sato, 1993). This contrasts with the organization of pathways from proprioceptors where direct connections with motor neurons are common (Eccles et al., 1957; Brown and Fyffe, 1981; Burrows, 1987; El Manira et al., 1991). In locusts, for example, the initial processing of the exteroceptive signals from trichoid sensilla is performed by spiking local interneurons. Each interneuron receives inputs from an array of these 
sensilla, which in part define its receptive field. Interneurons in one group with cell bodies at the ventral midline have receptive fields restricted to one hind leg (Burrows and Siegler, 1984, 1985), while those of a second group with cell bodies located more anteromedially have more extensive fields that can include other legs (Nagayama, 1990). The fields of the interneurons overlap so that one region of the leg is represented by several interneurons. The interneurons therefore preserve spatial information by mapping the surface of the leg as a series of overlapping receptive fields in a way that closely parallels the representation of a vertebrate leg in the receptive fields of certain spinal interneurons (Brown et al., 1980).

Tactile input to the CNS of insects is provided by two types of exteroceptors: trichoid sensilla (tactile hairs), which have been the subject of much analysis (Siegler and Burrows, 1983; Burrows, 1992), and basiconic sensilla. These two types of receptor differ in the length of their shafts that protrude from the surface of the cuticle, in the number of sensory neurons that innervate them, and in their distribution on a leg. The long trichoid sensilla are each innervated separately by a single sensory neuron and can be subdivided according to their different physiological responscs: high-threshold hairs respond phasically and adapt rapidly, and have a limited distribution; low-threshold hairs respond more tonically and continue to respond to repetitive stimuli, and are more common (Newland, 1991b). By contrast, the shorter basiconic sensilla are each innervated by five or six sensory neurons and serve a dual chemo- and mechanosensory function, because one sensory neuron is mechanosensory and the others chemosensory. They can respond to volatile odors (Dethier, 1972; Stadler and Hanson, 1975), but typically act as contact chemosensors. In locusts, they also form an elaborate mechanosensory system that is more sensitive than that formed by the surrounding trichoid sensilla (Newland and Burrows, 1994) and that should contribute substantially to the coding of tactile stimuli. In flies, the chemo- and the mechanosensory afferents project to different regions of neuropil, and this has led to the suggestion that each is processed by different sets of interneurons, while the mechanosensory signals from the basiconic and trichoid sensilla converge on the same interneurons (Murphey et al., 1989). In locusts, part of this prediction has been confirmed by the demonstration that the mechanosensory but not the chemosensory afferents from particular basiconic sensilla make direct, excitatory connections with local interneurons that are part of the local circuits controlling leg movements (Newland and Burrows, 1994).

In this article we address two issues about the processing by locust interneurons of exteroceptive signals from the two types of mechanoreceptors. First, we show that the mechanosensory afferents of the basiconic sensilla converge onto the same spiking local interneurons that process information from the mechanosensory afferents of adjacent trichoid sensilla. Second, we analyze the relative contribution of the inputs from the two types of receptors to the receptive fields of these interneurons.

\section{Materials and Methods}

Adult male and female locusts, Schistocerca gregaria (Forskål) were taken from our crowded laboratory culture and restrained ventral surface uppermost in Plasticine. The tibiae and tarsi of each leg were free to move. The left hind leg was rotated to allow access to particular arrays of exteroceptors on the femur, tibia, and tarsus according to the individual interneuron that was impaled in the central nervous system. The thorax was opened to expose the meso- and metathoracic ganglia, which were then stabilized on a wax-coated silver platform. The sheath covering the ventral midline of the metathoracic ganglion was treated with a $1 \%(\mathrm{w} / \mathrm{v})$ solution of protease (Sigma type XIV) for $1-2 \mathrm{~min}$ to facilitate the penetration of the somata of spiking local interneurons with glass microelectrodes filled with $2 \mathrm{M}$ potassium acetate. The thorax was perfused continuously with locust saline at $20-22^{\circ} \mathrm{C}$. The interneurons were characterized by their responses to mechanical or electrical stimulation of exteroceptors on one hind leg. The receptive field of an interneuron was first mapped approximately by touching arrays of receptors on different parts of a hind leg with a small paint brush. Regions evoking a depolarization or spikes in the impaled interneuron were then mapped more precisely by stimulating and recording from individual receptors of known type.

The spikes of the single afferent that innervates a trichoid sensillum were recorded by cutting its shaft and then placing a blunt, saline-filled microelectrode over the stump (Hodgson et al., 1955). The shaft of a basiconic sensillum has a pore at its tip so that a saline-filled recording trectrode placed over it directly records the afferent spikes. The electrodes were used to record afferent spikes and to stimulate the afferents electrically or mechanically by an imposed movement. A pair of hook electrodes was placed on $\mathrm{N} 5 \mathrm{r}$ ar the metathoracic ganglion so that the conduction velocities of the a. erents could be determined and the arrival time of their spikes at the edge of the ganglion predicted. The central projections of the afferents from these sensilla were stained by introducing $6 \%$ cobalt hexammine into the shafts of the sensilla (see Newland, 199 la, for full details). The shafts of the trichoid, but not the basiconic, sensilla were first cut and the cobalt was then allowed to diffuse for up to $10 \mathrm{~d}$ along the nerve and into the metathoracic ganglion. Despite these long diffusion times, only the basiconic sensilla on the proximal femur could be stained. The cobalt stain was then developed, enhanced with silver (Bacon and Altman, 1977), and the stained neurons drawn from whole-mounts of the ganglion or after it had been serially sectioned at $10 \mu \mathrm{m}$.

Recordings from the interneurons and the sensory neurons were stored on an FM tape recorder (Racal) for later display and analysis using a CED (Cambridge Electronic Design) interface and software, a digital oscilloscope and XY plotter, or a Gould ES1000 chart recorder. The results are based on recordings from 37 interneurons in 28 locusts. Detailed analyses of the receptive fields were restricted to three types of these interneurons with fields on the dorsal femur ( 10 locusts), ventral femur (10), and dorsal tibia and dorsal tarsus (12), respectively. For these experiments, a stable recording from the interneuron was required for as long as $2 \mathrm{hr}$. To control against changes in the electrode seal with the interneuron in these long recordings, and its consequent effects on the amplitudes of the synaptic potentials that were measured, the same sensilla were sampled at the start and end of an experiment. If the two recorded measures of the amplitudes of the potentials they evoked in the interneuron did not match, the experiment was discarded.

\section{Results}

\section{Types of exteroceptors}

Two main types of exteroceptors can be readily distinguished visually on the legs of a locust: trichoid sensilla (tactile hairs) have shafts that protrude $60-780 \mu \mathrm{m}$ from the cuticle, whereas the shafts of the basiconic sensilla are only 32-44 $\mu$ m (mean $38.4 \pm \mathrm{SD} 4.57, n=10$ ) (Fig. 1). Both types of receptor are intermingled over the surface of the leg, but their distribution is not uniform. For example, along a ventral ridge of the femur there are 15 trichoid and 15 basiconic sensilla, whereas on a dorsal row there are 19 trichoid but 26 basiconic sensilla $(n=$ 6 legs for all measurements). On the proximal dorsal tibia, basiconic sensilla outnumber the trichoid sensilla (28 to 6 ), but on the distal dorsal tibia the distribution is approximately reversed. The distribution of the receptors is such that both types will be stimulated if the leg makes contact with an object.

\section{Convergence onto local interneurons}

The mechanoreceptive afferents from both the trichoid sensilla and the basiconic sensilla converge onto spiking local interneurons of a ventral midline group (Fig. 1). Mechanical stimulation of an individual basiconic sensillum evoked a burst of spikes 

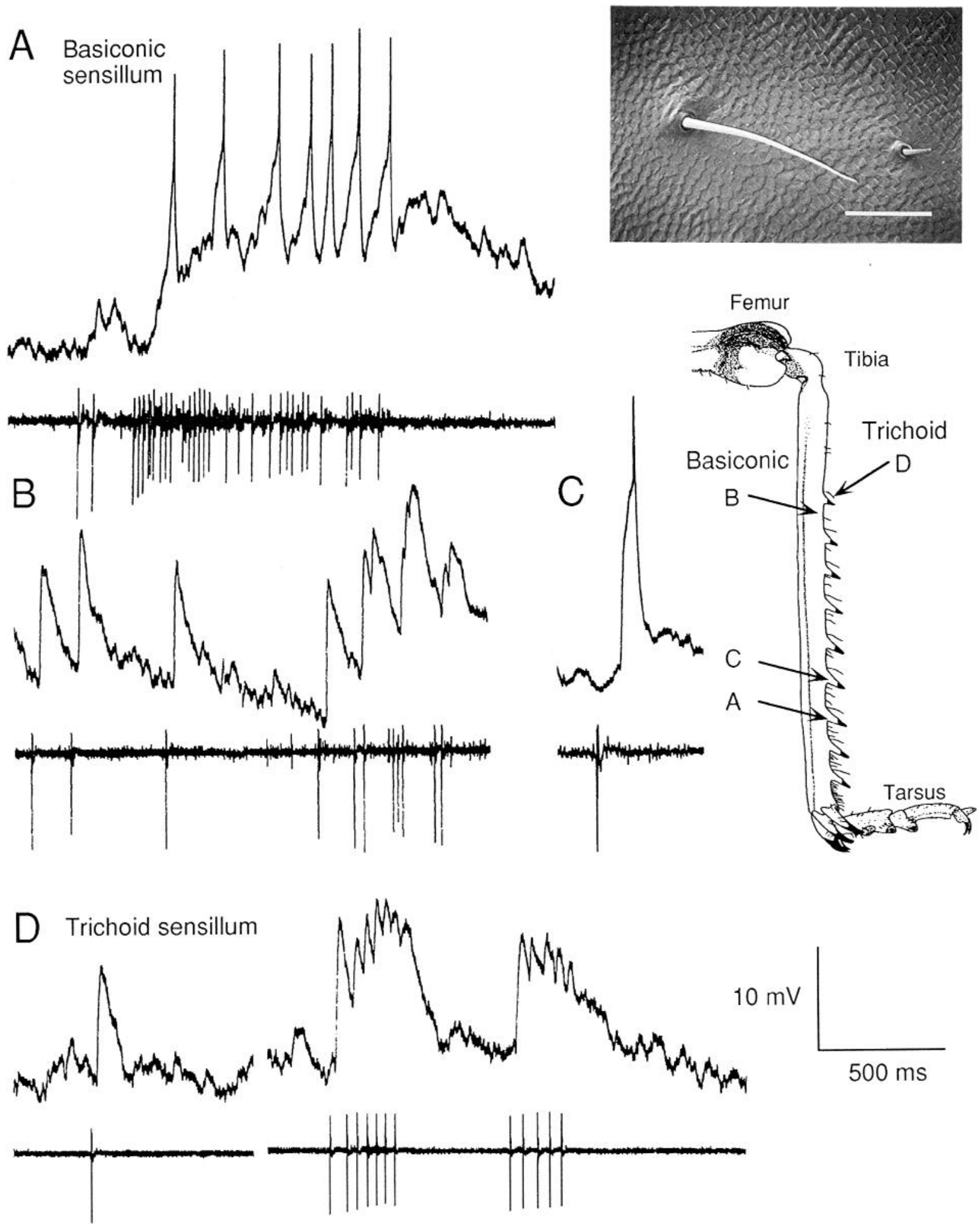

Figure 1. Mechanosensory afferents from basiconic and trichoid sensilla on the same region of the tibia both excite a spiking local interneuron of the midline group. $A-C$, Basiconic sensilla. $A, \mathrm{~A}$ burst of spikes in a mechanosensory afferent from a basiconic sensillum on the tibia evokes a burst of spikes in the interneuron. $B$, Each spike in the mechanosensory afferent, evoked by a movement of the recording electrode on another basiconic sensillum, is followed by an EPSP in the interneuron. The EPSPs sum but do not evoke spikes in the interneuron. $C$, The EPSP resulting from a single spike in an afferent from a third basiconic sensillum does evoke a spike in the interneuron. $D$, Trichoid sensilla. Each spike in an afferent from a trichoid sensillum on the tibia evokes an EPSP in the same interneuron. The EPSPs sum but do not evoke spikes in the interneuron. The positions of the sensilla in $A-D$ on the dorsal surface of the tibia are shown in the drawing. The scanning electron micrograph shows the morphology of the two types of sensilla on the dorsal femur. A trichoid sensillum is on the left and a basiconic sensillum is on the right. Scale bar, $100 \mu \mathrm{m}$. 


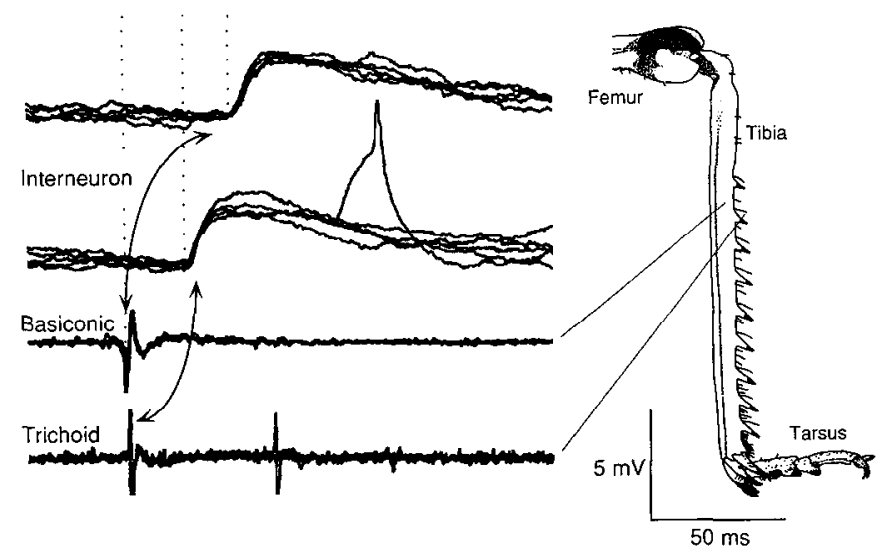

Figure 2. Mechanosensory afferents from adjacent basiconic and trichoid sensilla both connect with the same spiking local interneuron. Mechanosensitive afferents from two adjacent receptors, between the first and second spines on the tibia and $38 \mathrm{~mm}$ from the metathoracic ganglion, evoke consistent EPSPs in the interneuron. The EPSP from the trichoid sensillum occurs $33 \mathrm{msec}$ after the afferent spike but some $17 \mathrm{msec}$ before that of the basiconic sensillum. The afferent spikes were evoked by movement of the recording electrode on the sensillum. Four sweeps triggered by the afferent spikes are superimposed. The occurrences of the basiconic and trichoid afferent spikes are aligned although they were evoked sequentially. in its mechanosensory afferent followed by a depolarization and spikes in an interneuron in this group (Fig. $1 A$ ). Smaller movements evoked afferent spikes of lower frequency, each of which was followed consistently by a depolarizing synaptic potential in the interneuron (Fig. 1B). A single afferent spike from a particular basiconic sensillum could evoke a synaptic potential that led to a spike in the interneuron (Fig. $1 C$ ). The synaptic potentials are therefore EPSPs (Newland and Burrows, 1994).

Mechanical stimulation of trichoid sensilla on the same region of the tibia as these basiconic sensilla also evoked EPSPs in the same interneuron (Fig. 1D). The afferent spikes were followed consistently by EPSPs that summed, but in this example did not exceed the spike threshold of the interneuron (Fig. 1D). On other occasions, this and afferents from other trichoid sensilla on the tibia could evoke spikes in this interneuron. The potentials evoked by these afferents are also EPSPs (Siegler and Burrows, 1983).

The physiological evidence suggests that the mechanosensory afferents from both the basiconic and trichoid sensilla synapse directly on the same midline interneurons. The EPSP croked by the mechanosensory afferent from a basiconic sensillum on the tibia followed the sensory spike with a constant latency (Fig. 2). Similarly, the EPSP from an adjacent trichoid sensillum also occurred with a constant, but much shorter latency (Fig. 2). This difference in latency is explained by the different conduction
Figure 3. Central projections of afferents from basiconic and trichoid sensilla. $A$, Central projections of the afferents from basiconic sensilla on the proximal dorsal femur seen in a wholemount (i) or in transverse section (ii). $B$, The single afferent from one trichoid sensillum at a similar position on the femur, again viewed in whole-mount $(i)$ and in transverse section (ii). The right half of the metathoracic ganglion is shown with the boundary of the neuropil indicated by dashed lines and lateral nerves $l-3$ and 5, 6 labeled. Some of the prominent longitudinal tracts and commissures are shown in the sections. $D C I I I$, dorsal commissure III; DIT, dorsal intermediate tract; $D M T$, dorsal median tract; $L D T$, lateral dorsal tract: $M D T$, median dorsal tract; $M V T$, median ventral tract; $V I T$, ventral intermediate tract; $V M T$, ventral median tract; $I V A C$, lateral ventral association center; $v V A C$, ventralmost ventral association center.

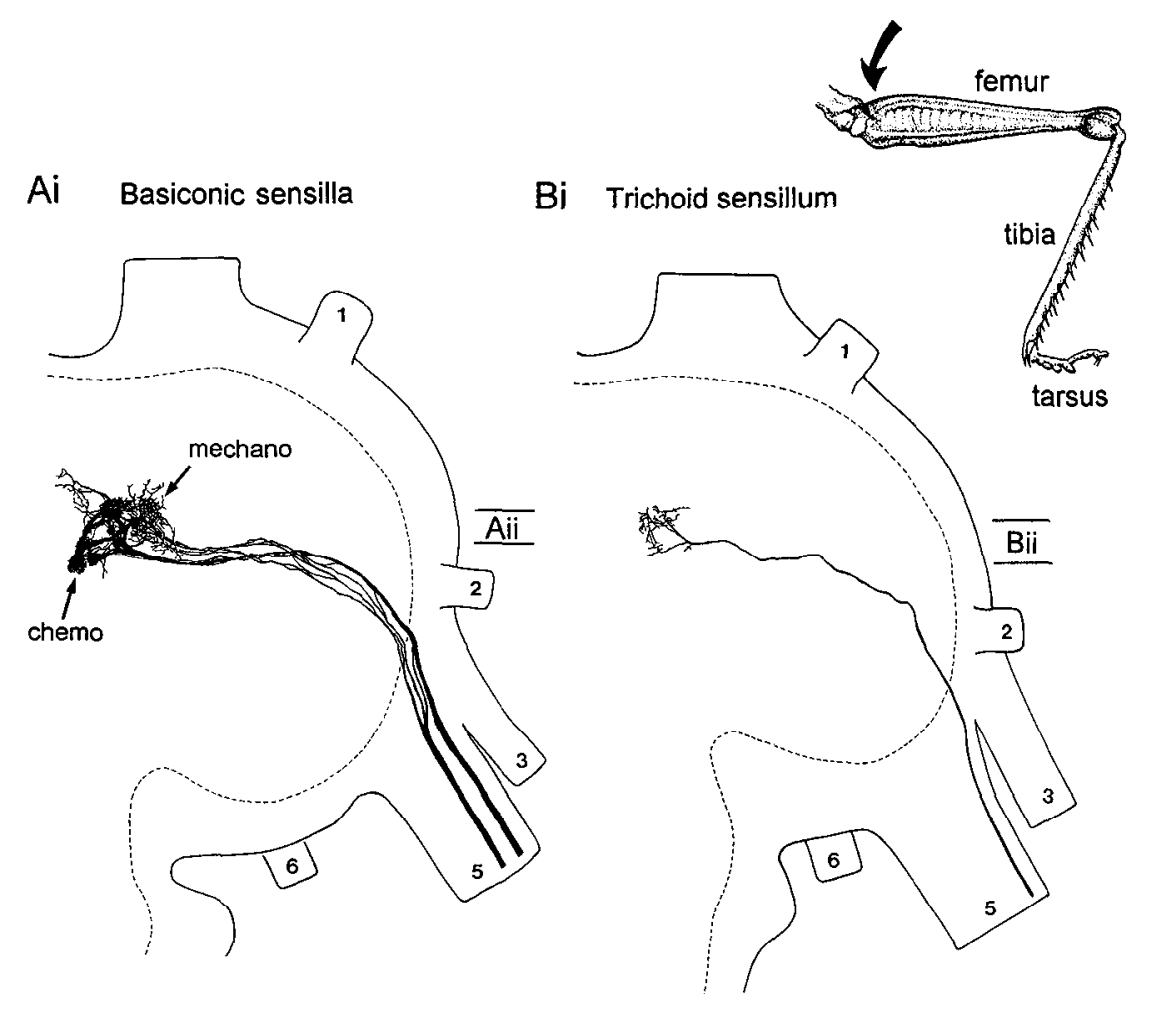

Aii

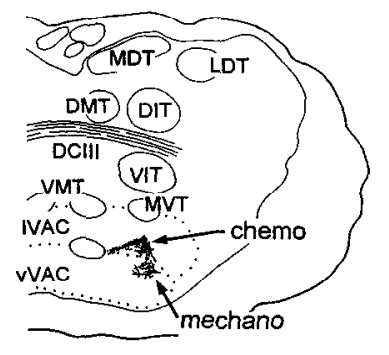

Bii

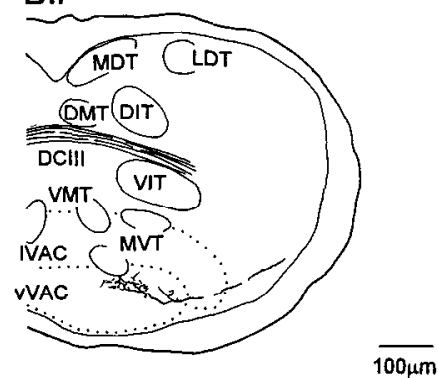


velocities of the afferents from the two types of receptor; basiconic afferents had conduction velocities of $0.99 \mathrm{~m} \cdot \mathrm{sec}^{-1}$ (SE

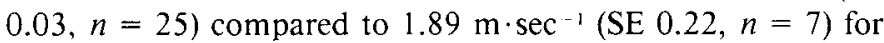
the trichoid afferents. For both types of afferents, the central synaptic delay was less than $2 \mathrm{msec}$, in which time the spikes must have traveled from the edge of the ganglion to the synaptic sites and evoked a synaptic potential in the interneuron. This central delay would be sufficient for there to be only one synapse in the pathway.

The anatomy of the afferents from the basiconic and the trichoid sensilla, at the level of the light microscope, suggests that they project to the ventral association center (Fig. 3A). Moreover, receptors from the same region of the leg project to the same area within this region of neuropil so that they could contact the same branching region of specific interneurons. For example, cobalt stains of a group of basiconic sensilla on the proximal, dorsal femur revealed two populations of fibers that both projected to the same area of neuropil as seen in wholemounts of the metathoracic ganglion (Fig. 3Ai). Many of the fibers were extremely fine, formed glomerular endings, and are presumed to represent the chemosensitive afferents. The fewer thicker fibers formed projections similar to those of the trichoid afferents and are presumed to represent the mechanosensory afferents from the basiconic sensilla. These fibers are nevertheless still of smaller diameter than those of the trichoid afferents, a factor that is also reflected in the slower conduction velocities of their spikes. In transverse sections, the thicker fibers projected to the ventralmost ventral association center, whereas the finer fibers projected slightly more dorsally to the lateral ventral association center (Fig. 3Aii). The afferent from an adjacent trichoid sensillum projected to the same area of neuropil as the presumed mechanosensory afferents from the basiconic sensilla, as seen in whole-mounts and transverse sections (Fig. 3Bi,ii).

\section{Summation of inputs from different types of receptors}

The apparently direct connections made with specific interneurons by the two types of receptors and the spatial overlap of their branches suggested that the synaptic inputs from adjacent receptors might interact and summate. To test this, one electrode was placed on a basiconic sensillum and a second on a nearby trichoid sensillum while recordings were made from a midline spiking local interneuron (Fig. 4). A single spike in either the mechanosensory afferent from the basiconic sensillum (Fig. $4 A$ ) or from the trichoid sensillum (Fig. $4 B$ ) evoked an EPSP but not a spike in the interncuron. When a simultancous mcchanical stimulus to both receptors evoked a spike in each afferent, then their resulting inputs to the interneuron summed to evoke a spike (Fig. $4 C$ ). The summation between the two afferents was more clearly seen when an electrical stimulus was applied to each receptor in sequence and the interval between the two stimuli gradually reduced (Fig. $4 D-G$ ). The different conduction velocities of the two afferents always ensured that there was an additional and approximately $17 \mathrm{msec}$ latency between the evoked spikes by the time they reached the metathoracic ganglion. At intervals of $500 \mathrm{msec}$ the two EPSPs did not sum (Fig. 4D), but at intervals of $100 \mathrm{msec}$ or less summation did occur (Fig. $4 E, F$ ). At intervals of $20 \mathrm{msec}$ the summation was sufficient to evoke a spike in the interneuron (Fig. $4 G$ ).

\section{Organization of receptive fields of interneurons}

Each interneuron is excited by a particular array of basiconic and trichoid sensilla, so its receptive field can be described according to the distribution of these receptors on different regions

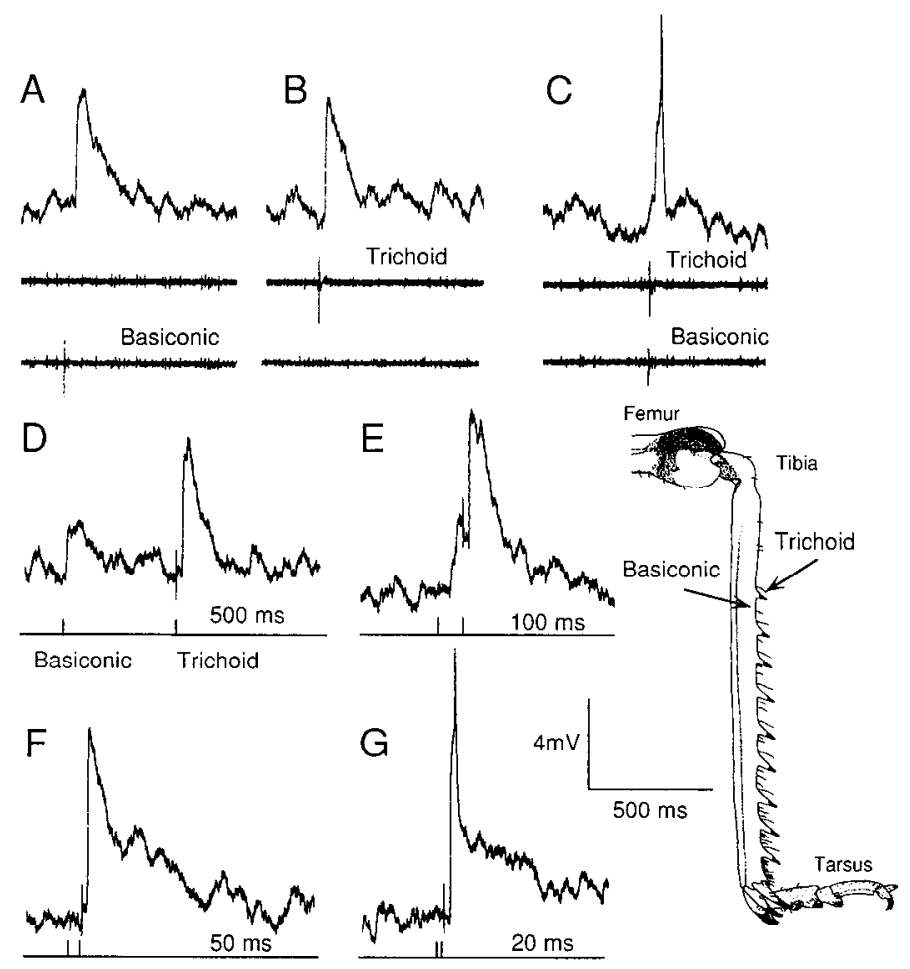

Figure 4. Summation of the synaptic potentials evoked in a spiking local interneuron by mechanosensory afferents from a basiconic and an adjacent trichoid sensillum. $A$ and $B$, Individual spikes in the mechanosensory afferent from either a basiconic $(A)$ or a trichoid $(B)$ sensillum evoke EPSPs that do not give rise to spikes in the interneuron. $C$, When the afferent spikes from both receptors occur at the same time, as a result of a simultaneous mechanical stimulus, the EPSPs sum to make the interneuron spike. The sensory spikes (lower traces) are recorded by electrodes on the individual receptors. $D-G$, The basiconic sensillum and then the trichoid sensillum are stimulated electrically with single shocks at progressively shorter intervals. The EPSPs progressively sum and at the shortest intervals tested evoked a spike in the interneuron. The positions of the sensilla on the proximal tibia are indicated in the drawing of the hind leg.

of a hind leg. The following experiments were therefore undertaken with two objectives: first, to determine if the two types of receptor contributing to the receptive field of an interneuron had the same spatial distribution; sccond, to detcrmine the relative contribution of each receptor to the excitation of an interneuron. The receptive fields of nine types of interneuron, probably representing nine individual interneurons, were plotted by mechanically stimulating arrays of receptors with a fine paint brush (Fig. 5). Such stimuli excited groups of both basiconic and trichoid sensilla. This coarse sampling of the receptive fields showed that they consisted of one contiguous and overlapping array of receptors on specific regions of the leg. Three interneurons, each recorded in at least 10 locusts, were then analyzed in detail by stimulating individual receptors mechanically and recording the EPSPs they generated in an interneuron.

The basiconic and trichoid sensilla contributing to the receptive field of the interneuron in Figure 6 were intermingled on the dorsal surface of the tibia (the surface that bears the spines) and on the dorsal surface of the tarsus. Receptors elsewhere on the leg, including the other surfaces of the tibia and tarsus, provided no input to this interneuron. The boundary of a receptive field could therefore be sharp. Within the receptive field, 


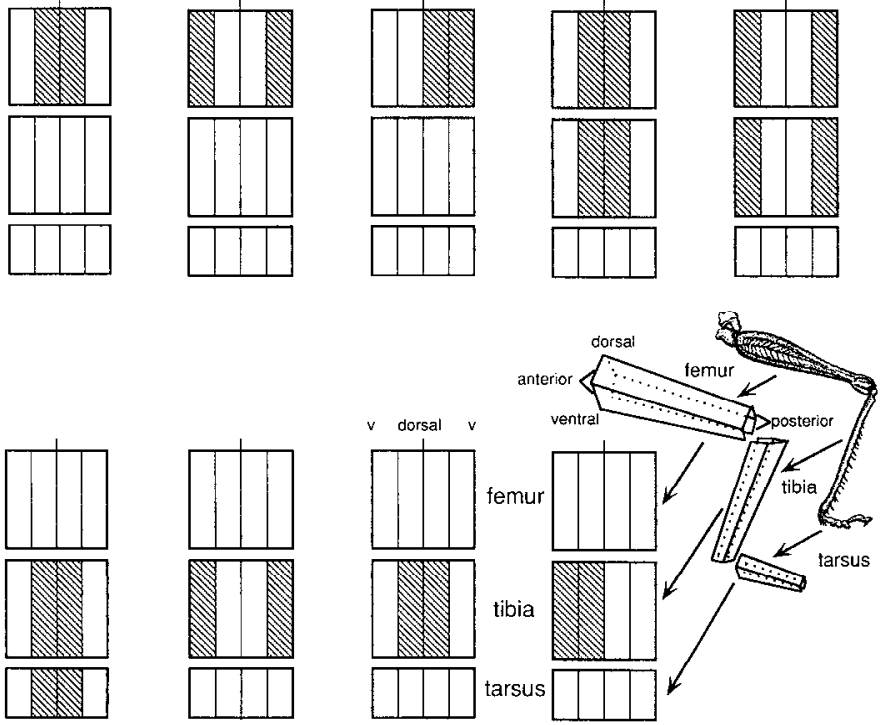

Figure 5. Summary of the receptive fields of nine spiking local interneurons with inputs from trichoid and basiconic sensilla that have been studied here. The femur, tibia, and tarsus of a hind leg are represented diagrammatically by three rectangles divided along the ventral midline into dorsal and ventral surfaces (see inset drawings). The region of the leg representing the receptive field of an interneuron is hatched.

the mechanosensory afferents of the basiconic sensilla produced EPSPs with different amplitudes, and differed in their ability to make the interneuron spike. The largest-amplitude EPSPs were generated by basiconic sensilla between spines 6 and 7; individual spikes in these afferents sometimes led directly to spikes in the interneuron. Sensilla located more distally on the tibia generated smaller-amplitude EPSPs, whereas those on the tarsus generated EPSPs that were only half the amplitude of the largest ones. The amplitude of the EPSPs generated by the more proximal sensilla on the tibia were also smaller, but those close to the femorotibial joint still generated EPSPs that were almost $75 \%$ of the amplitude of the most effective sensillum.

The trichoid afferents also evoked EPSPs of differing amplitude in the interneuron according to the position of the receptors on the tibia (Fig. 6). The largest EPSPs were generated by trichoid sensilla just proximal to the first spinc. The afferents from more distal trichoid sensilla generated EPSPs of progressively smaller amplitude, but those of the long trichoid sensillum at the eighth spine still evoked EPSPs that were $50 \%$ of the amplitude of the largest. More proximal trichoid sensilla also generated smaller-amplitude EPSPs, and as for the basiconic sensilla, none on the femur generated an input. The most effective trichoid sensilla were thus spatially separated from the most effective basiconic sensilla, but gradients in the amplitudes of the EPSPs were similar for both types of receptors.

In other interneurons, the basiconic and trichoid sensilla that generated the largest EPSPs were adjacent to each other. For example, in the interneuron of Figure 7, which had a receptive field on the dorsal femur, the largest EPSPs were generated by adjacent basiconic and trichoid sensilla dorsal to the semilunar processes associated with the femorotibial joint. Spikes in the afferents from individual reccptors in this region could sum to evoke spikes in the interneuron. From these receptors, gradients of less effective receptors extended both distally and proximally. The gradient was steep distally, such that receptors at the end

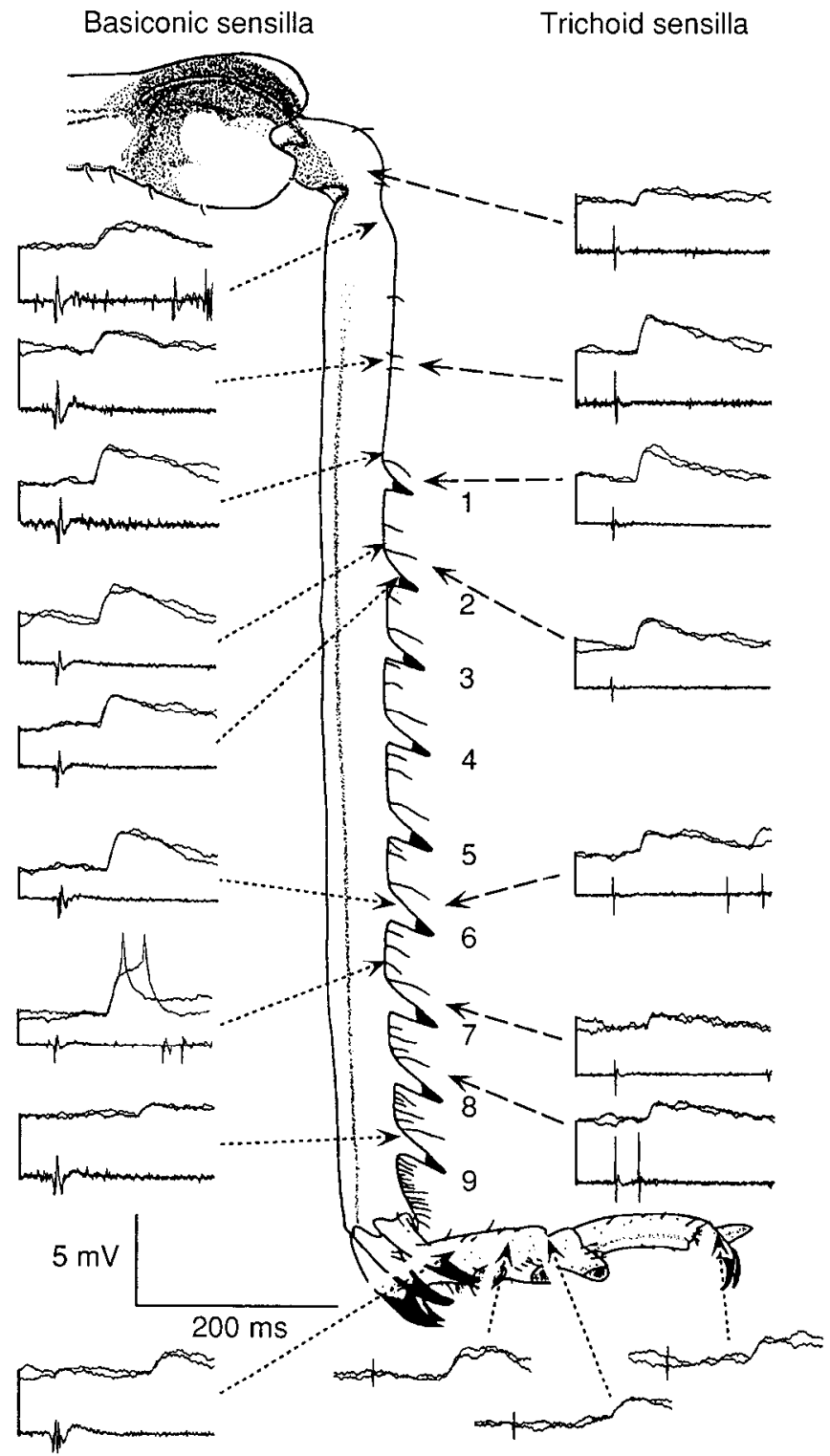

Figure 6. Receptive field of a spiking local interneuron with inputs from basiconic and trichoid sensilla on the dorsal tibia and dorsal tarsus. The largest-amplitude EPSPs, and those most likely to evoke spikes in the interneuron, are from basiconic sensilla on the middle region of the tibia. The amplitude of the EPSPs declines both proximally and distally along the tibia. The EPSPs generated by the trichoid sensilla show similar gradients in amplitude. Twenty-five basiconic and 17 trichoid sensilla were sampled, from a larger population that comprise the complete receptive field, but only 12 and 7 , respectively, are shown from all parts of the field. In this and subsequent figures each trace consists of superimposed sweeps triggered by the afferent spikes evoked by movement of the recording electrode. The basiconic sensilla on the tarsus were stimulated electrically and the sweeps were triggered by the stimulus artifacts. The positions of the basiconic sensilla are indicated by the dotted lines and those of the trichoid sensilla by the dashed lines. The rigid spines on the dorsal surface of the tibia are numbered.

of the femur, less than $1 \mathrm{~mm}$ away from the most effective receptors, evoked EPSPs that were only $15 \%$ of the amplitude of the largest. Proximally, the gradient was more gradual, so that even the most proximal receptors on the dorsal surface evoked EPSPs that were $40 \%$ of the largest. The gradients for both the basiconic and trichoid sensilla were the same along the 


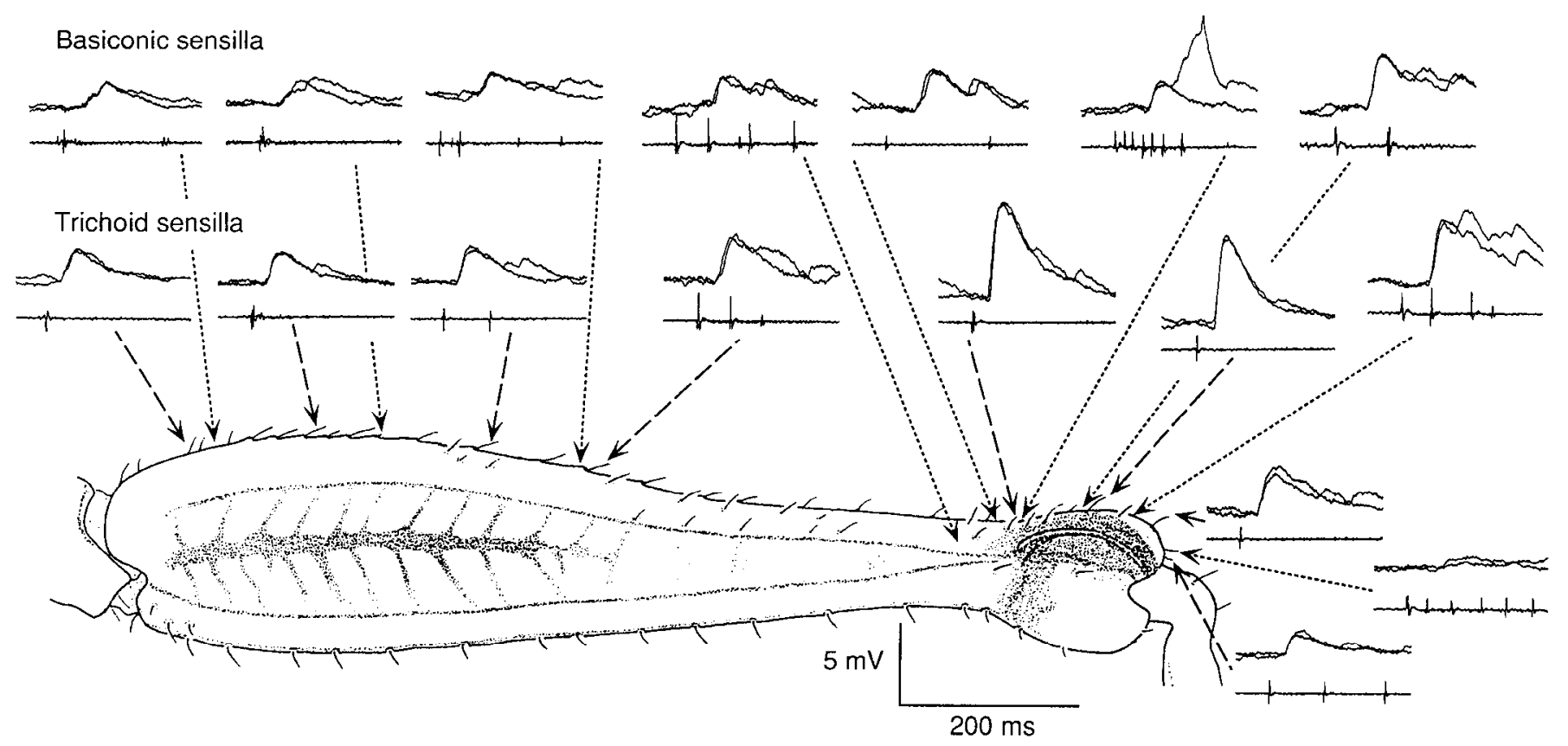

Figure 7. Receptive field of a spiking local interneuron with inputs from basiconic and trichoid sensilla on the dorsal surface of the femur. The effectiveness of the inputs from the two types of sensilla show the same gradients: the most effective inputs are from sensilla dorsal and proximal to the semilunar process close to the femorotibial joint. Seventeen basiconic and 11 trichoid sensilla were sampled but only nine and eight, respectively, are shown.

whole length of the femur, but the EPSPs evoked by the trichoid sensilla were always larger.

The mapping of the receptors onto specific interneurons and the similarity in the effects caused by individual receptors are remarkably constant from animal to animal (Fig. 8A, $B$ ). One interneuron with a receptive field on the ventral femur was recorded in 10 animals and analyzed in detail in two. Gradients in the amplitudes of the EPSPs generated by the receptors were similar in both examples. The most effective basiconic and trichoid sensilla both occurred on the same distal region of the femur. From this region there was a gradual and progressive decline in the amplitude of the EPSPs generated by both more distal and more proximal sensilla (Fig. $8 A, B$ ). Although the overall gradients in the amplitude of evoked EPSPs were smooth, some individual receptors gave either larger or smaller potentials than was predicted from their position. For example, one basiconic sensillum in Figure $8 B$ (labeled d) evoked an EPSP of larger amplitude than either the next distal or the next proximal receptor. Similarly, when three basiconic sensilla occurred together in the row between two trichoid sensilla, the gradient in the amplitude of their evoked EPSPs was not always obeyed. In Figure $8 A$, the most distal receptor (c) of three sensilla (a-c) generated the largest-amplitude EPSP, whereas in Figure $8 B$ it was the middle one (f). The trichoid sensilla typically evoked a larger-amplitude EPSP in the interneuron than did adjacent basiconic sensilla. Occasionally, however, a basiconic sensillum was the most effective (d in Fig. 8B). In other interneurons, the basiconic sensilla evoked larger-amplitude EPSPs than the trichoid sensilla.

\section{Discussion}

\section{Convergence in the CNS}

This article has shown that the mechanosensory information from the two types of exteroceptors on a locust hind leg con- verges onto the same local interneurons of a ventral midline group in a manner that accords with their position on the leg. The conncctions from both appcar to be dircet and mediatcd by the release of a chemical transmitter (Siegler and Burrows, 1983; Newland and Burrows, 1994). These spiking local interneurons also receive inputs that are apparently direct from campaniform sensilla (Siegler and Burrows, 1983) that respond to deformations of the cuticle, caused either by external objects or by contractions of the muscles. The result is that exteroceptive information from particular spatial arrays of receptors is brought together and processed by the same interneurons. Moreover, this information can, in some interneurons, be merged with proprioceptive signals from specific joints (Burrows, 1985). Other interneurons respond exclusively to either tactile or proprioceptive inputs, so the mechanosensory information can be processed in separate, parallel channels (Burrows, 1985; Burrows and Newland, 1993). The midline spiking interneurons are, however, not the only neurons to process the mechanosensory signals. The signals from all the different types of receptor examined so far are processed in parallel by nonspiking interneurons, by other spiking local interneurons, and by projection interneurons (Burrows, 1989). A few exteroceptive (Laurent and Hustert, 1988) and many proprioceptive afferents (Burrows, 1987) also synapse directly on the motor neurons. A reasonable expectation from these findings would be that the mechanosensory afferents from the basiconic sensilla also make many divergent connections with central neurons.

\section{Receptive fields}

The contribution of individual basiconic and trichoid receptors to the excitation of an interneuron is not uniform across a receptive field. The mechanosensory afferents from some receptors evoke large EPSPs that by themselves are able to evoke spikes in the interneuron. The gain of the synaptic connection 
A

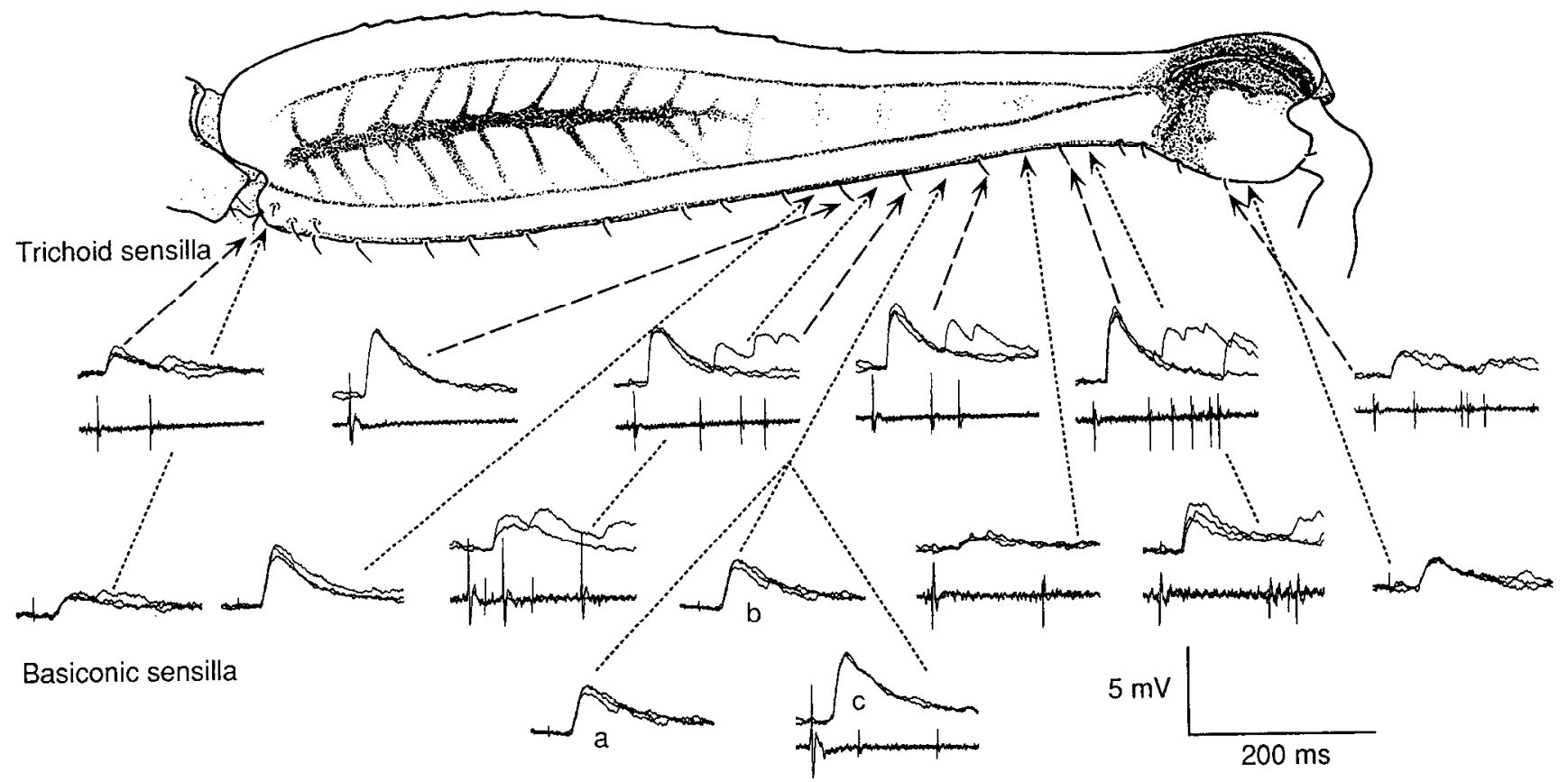

B

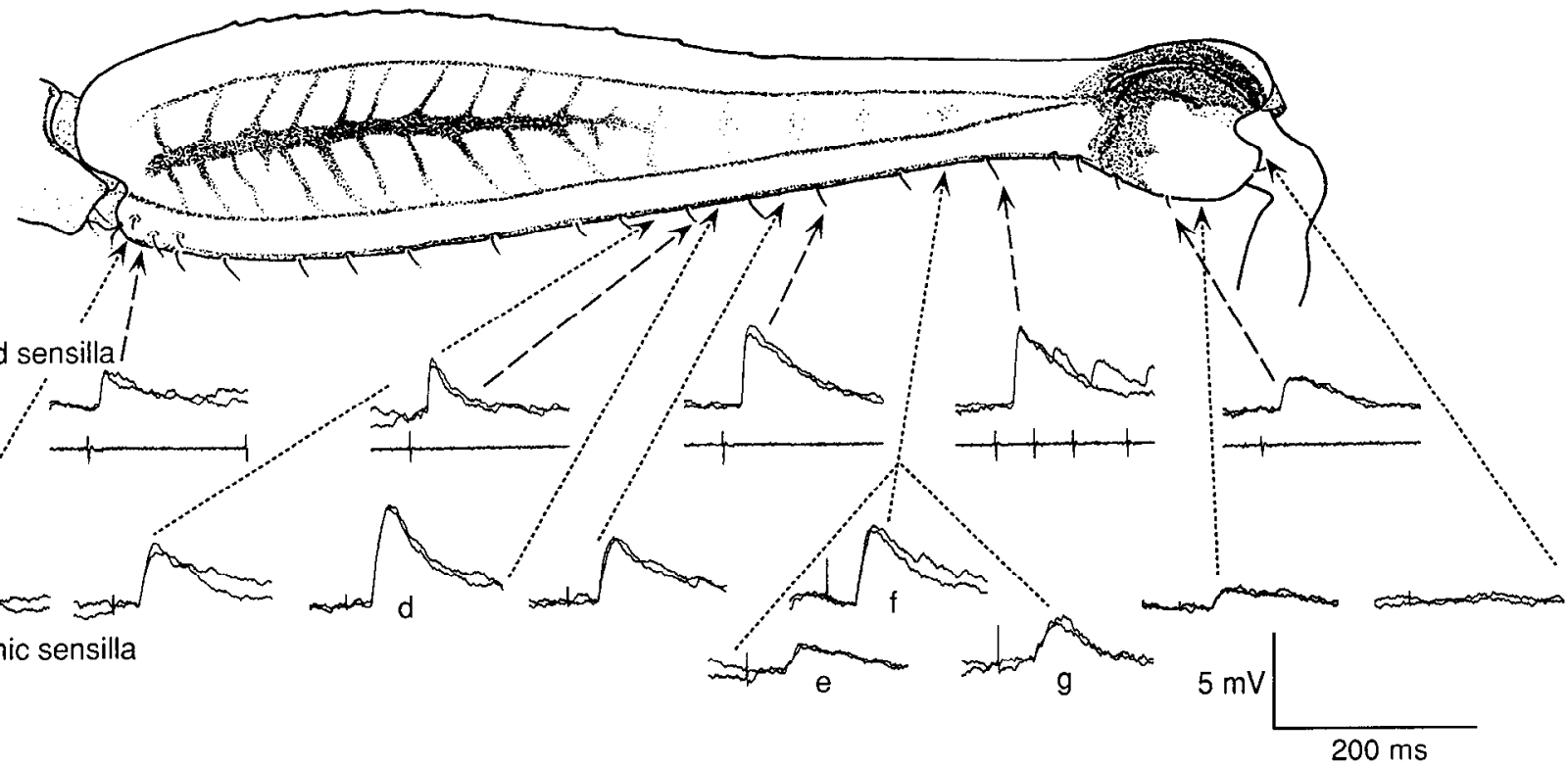

Figure 8. Receptive fields of a spiking local interneuron with inputs from basiconic and trichoid sensilla on the ventral surface of the femur. The same interneuron was recorded in two locusts $(A, B) . A$, The most effective inputs are from sensilla proximal to the semilunar process, with the effectiveness of the other sensilla declining both proximally and distally. $B$, The receptive field of the interneuron in a second locust shows the precision with which the organization of the receptive field is specified in different animals. Some of the basiconic sensilla were stimulated electrically and the sweeps triggered from the stimulus artifacts. 
from these afferents is therefore high, the transmission reliable, and sensory information is preserved for the next stages in the processing. Other receptors evoke EPSPs of smaller amplitude, and are typically arranged in spatial gradients radiating from those that generate the largest EPSPs. These gradients are aligned with the axes of the legs and mean that a receptive field has just one area of receptors of a particular type with the highest synaptic gain. The gradients can be steep at the edges of a field such that the boundaries are sharp. For the trichoid afferents (Burrows, 1992), and probably also for the basiconic afferents, the amplitude of the EPSPs equates with the gain of the synaptic connection defined by the probability with which a single sensory spike will evoke a spike in the interneuron. The gain is determined by the position of the receptor on the leg.

The gradients in the amplitudes of the evoked EPSPs, and in their ability to make an interneuron spike, are similar within a particular interneuron for both the trichoid sensilla and the basiconic sensilla. Often the most effective trichoid sensilla and basiconic sensilla are contiguous, though in some interneurons they can be slightly shifted from one another. This suggests that the same developmental and organizational constraints determine the establishment of the connections of both types of receptor. The terminals of the afferents from trichoid sensilla form a three-dimensional representation of the surface of the leg in a ventral region of neuropil (Newland, 1991a). So far, we know only that the projections from basiconic sensilla on the proximal femur are in accord with this map as they are in the same region of ventral neuropil as those from adjacent trichoid sensilla. The similar gradients in the amplitudes of the EPSPs generated by the trichoid and basiconic sensilla could result from a similar spatial distribution of their synapses on an interneuron, or from differences in the number of release sites and the number of quanta released at each site.

\section{Organization of exteroceptive signal processing}

It is now possible to add the results presented here to a definition of the organizational features for the processing of tactile information from a hind leg, and for the structure of the receptive fields of the midline spiking local interneurons. These features can be summarized as follows:

(1) Interneurons receive an apparently direct input from the mechanosensory afferents that inncrvatc both basiconic and trichoid sensilla.

(2) The two types of receptors providing input to a particular interneuron are intermingled and form one contiguous array on specific regions of a hind leg. No examples have been found of regions of the leg where trichoid but not basiconic sensilla, or vice versa, provide input to an interneuron.

(3) Not all receptors within the boundary of a receptive field contribute inputs to an interneuron. The most notable specialization is that in some fields the high-but not the low-threshold trichoid sensilla may contribute (Burrows, 1992). No comparable differences have been found in the response properties of the basiconic sensilla (Newland and Burrows, 1994).

(4) Not all the trichoid sensilla and not all the basiconic sensilla within a receptive field contribute equally to the excitation of an interneuron. Typically, one array of trichoid sensilla and onc array of basiconic sensilla provide the most effective inputs, whereas surrounding receptors are less effective. In the majority of interneurons the most effective trichoid sensilla and the most effective basiconic sensilla are adjacent. In different interneu- rons, either particular basiconic sensilla or particular trichoid sensilla provide the most powerful input.

(5) The organization of the receptive field of a particular interneuron, identified by its morphology, in different animals is remarkably similar in the relative contribution of individual basiconic and trichoid receptors, and indicates considerable specificity in the establishment of the connections.

(6) The delay between the information arriving at the CNS from adjacent receptors of the two types results from the markedly different conduction velocities of their spikes, and can be a significant proportion of the time taken to perform a tactile reflex.

\section{Functional consequences of this organization}

Why should there be two distinct types of exteroceptors providing information about tactile stimuli? The basiconic sensilla are short, low-angular-threshold receptors, whereas the trichoid sensilla are longer but have higher thresholds (Newland, $1991 \mathrm{~b}$; Newland and Burrows, 1994). This means that the basiconic sensilla are more sensitive because their shafts need to be deflected through a smaller angle to make the mechanosensory afferent spike. An approaching object will therefore first stimulate the long, but high-threshold trichoid sensilla before finally stimulating the short, low-threshold basiconic sensilla. We do not know whether the stimulation of the different receptors can be discriminated but only that the inputs from both are summed by the spiking local interneurons and should increase sensitivity. Some stimuli may therefore deflect only the trichoid sensilla, which because of their high threshold, may not evoke a sufficient sensory response to make the local interneurons spike. Other stimuli that contact the cuticle will deflect both the trichoid and basiconic sensilla. The higher sensitivity of the basiconic sensilla, and the typically higher density of these receptors in some regions, will ensure that a larger sensory discharge is evoked that will be summed by the interneurons and make them spike. Moreover, the excitation may be further increased by the parallel pathways activated by the basiconic sensilla that result in a prolonged depolarization of the spiking local interneurons (Newland and Burrows, 1994).

The basiconic sensilla also serve a second role as contact chemoreceptors, and may even respond to volatile odors. There is much evidence to indicate that the high density of chemoreceptors on the feet is used by many insects in food discrimination and host plant selection (Dethier, 1976). Furthermore, locusts will wave their legs if the tarsi contact certain chemicals (White and Chapman, 1990). If the basiconic sensilla are to act as contact chemoreceptors and mediate directed and adaptive movements of the legs, then it would seem essential that the spatial location of the stimulus is preserved. The processing by the local interneurons described here maintains that spatial information and could therefore be used to activate specific pathways that result in a directed motor response. When the basiconic receptors respond to odors, the requirement for spatial information is less obvious. Challenging a leg with odors from certain heavy oils such as eucalyptus oil, however, results in the leg being withdrawn from the source of the odor (Slifer, 1954, 1956) in movements that resemble those caused by mechanical stimulation of the exteroceptors (Siegler and Burrows, 1986). In flies, the chemosensory afferents project to different regions of neuropil from the mechanosensory afferents (Murphey et al., 1989). In locusts, the mechano- and chemosensitive afferents 
also appear to project to different regions, and the chemosensitive afferents do not synapse on the midline spiking interneurons that receive direct inputs from the mechanosensory afferents (Newland and Burrows, 1994). This implies that the initial processing of the chemo- and mechanosensory signals is performed in different channels, but the behavior indicates that the two modalities must converge if similar local responses of a leg are to be produced. Locating where the convergence of these two modalities occurs is the next task in the analysis of these local circuits for the control of leg movements.

\section{References}

Bacon JP, Altman JS (1977) A silver intensification method for cobaltfilled neurones in wholemount preparations. Brain Res 138:359-363.

Brown AG, Fyffe REW (1981) Direct observations on the contacts made between Ia afferent fibres and alpha motoneurones in the cat's lumbrosacral spinal cord. J Physiol (Lond) 313:121-140.

Brown AG, Fyffe REW, Noble R, Rose PK, Snow PJ (1980) The density, distribution and topographical organization of spinocervical tract neurones in the cat. J Physiol (Lond) 300:409 428.

Brown AG, Koerber HR, Noble R (1987) An intracellular study of spinocervical tract cell responses to natural stimuli and single hair afferent fibres in cats. J Physiol (Lond) 382:331-354.

Brown PB, Gladfelter WE, Culberson JC, Covalt-Dunning D, Sonty RV, Pubols LM, Millecchia RJ (1991) Somatotopic organization of single primary afferent axon projections to cat spinal cord dorsal horn. J Neurosci 1 1:298-309.

Burrows M (1985) The processing of mechanosensory information by spiking local interneurones in the locust. J Neurophysiol 54:463-478.

Burrows M (1987) Parallel processing of proprioceptive signals by spiking local interneurones and motor neurones in the locust. J Neurosci 7:1064-1080.

Burrows M (1989) Processing of mechanosensory signals in local reflex pathways of the locust. J Exp Biol 146:209-227.

Burrows M (1992) Reliability and effectiveness of transmission from exteroceptive sensory neurons and spiking local interneurons in the locust. J Neurosci 12:1477 1489.

Burrows M, Newland PL (1993) Correlation between the receptive fields of locust interneurons, their dendritic morphology, and the central projections of mechanosensory neurons. J Comp Neurol 329: $412-426$.

Burrows M, Siegler MVS (1984) The morphological diversity and receptive fields of spiking local interneurones in the locust metathoracic ganglion. J Comp Neurol 224:483-508.

Burrows M, Siegler MVS (1985) The organization of receptive fields of spiking local interneurones in the locust with inputs from hair afferents. J Neurophysiol 53:1147-1157.

Dethier VG (1972) Sensitivity of the contact chemoreceptors of the blowfly to vapours. Proc Natl Acad Sci USA 69:2189-2192.

Dethier VG (1976) The hungry fly. Cambridge, MA: Harvard UP.

Eccles JC, Eccles RM, Lundberg A (1957) Synaptic actions on motoneurones in relation to the two components of the group I muscle afferent volley. J Physiol (Lond) 136:527-546.

El Manira A, Cattaert D, Clarac F (1991) Monosynaptic connections mediate resistance reflex in crayfish (Procambarus clarkii) walking legs. J Comp Physiol [A] 168:337-349

Hodgson ES, Lettvin JY, Roeder KD (1955) Physiology of a primary chemoreceptor unit. Science 122:417-418.

Johnson SE, Murphey RK (1985) The afferent projection of mesothoracic bristle hairs in the cricket, Acheta domesticus. J Comp Physiol [A] 156:369-379.

Kent KS, Levine RB (1988) Neural control of leg movements in a metamorphic insect: sensory and motor elements of the larval thoracic legs in Manduca sexta. J Comp Neurol 271:559-576.

Koerber HR, Brown PB (1982) Somatotopic organization of hindlimb cutaneous nerve projections to cat dorsal horn. J Neurosci $48: 481-$ 489.

Laurent GJ, Hustert R (1988) Motor neuronal receptive fields delimit patterns of activity during locomotion of the locust. J Neurosci 8:43494366.

Levine RB, Pak C, Linn D (1985) The structure, function and metamorphic reorganisation of somatotopically projecting sensory neurons of Manduca sexta larvae. J Comp Physiol [A] 157:1-13.

Light AR, Perl ER (1979) Spinal terminations of functionally identified primary afferent neurons with slowly conducted myelinated fibres. J Comp Neurol 186:133-150.

Merritt DI, Murphey RK (1992) Projections of leg proprioceptors within the CNS of the fly Phormia in relation to the generalized insect ganglion. J Comp Neurol 322:16-34.

Murphey RK, Jacklet A, Schuster L (1980) A topographic map of sensory cell terminal arborizations in the cricket CNS: correlation with birthday and position in a sensory array. J Comp Neurol 191: 53-64.

Murphey RK, Possidente D, Pollack G, Merritt DJ (1989) Modalityspecific axonal projections in the CNS of the flies Phormia and Drosophila. J Comp Neurol 290:185-200.

Nagayama T (1990) The organisation of receptive fields of an anteromedial group of spiking local interneurones in the locust with exteroceptive inputs from the legs. J Comp Physiol [A] 166:471-476.

Nagayama T, Sato $M$ (1993) The organisation of exteroceptive information from the uropod to ascending interneurons of the crayfish. $J$ Comp Physiol [A] 172:281-294.

Newland PL (1991a) Morphology and somatotopic organisation of the central projections of afferents from tactile hairs on the hind leg of the locust. J Comp Neurol 312:493-508.

Newland PL (1991b) Physiological properties of afferents from tactile hairs on the hindlegs of the locust. J Exp Biol 155:487-503.

Newland PL, Burrows M (1994) Processing of mechanosensory information from gustatory receptors on a hind leg of the locust. J Comp Physiol $[\mathrm{A}]$, in press.

Nyberg G, Blomqvist A (1985) Somatotopic organization of the forelimb cutaneous nerves in brachial dorsal horn: an anatomical study in the cat. J Comp Neurol 242:28-39.

Peterson BA, Weeks JC (1988) Somatotopic mapping of sensory neurons innervating mechanosensory hairs on the larval prolegs of $\mathrm{Man}$ duca sexta. J Comp Neurol 275:128-144.

Pflüger HJ, Braunig P, Hustert R (1981) Distribution and specific central projections of mechanoreceptors in the thorax and proximal leg joints of locusts. II. The external mechanoreceptors: hair plates and tactile hairs. Cell Tissue Res 216:79-96.

Shortland P, Woolf LJ, Fitzgerald M (1989) Morphology and somatotopic organization of the central terminals of hindlimb hair follicle afferents in rat lumbar spinal cord. J Comp Neurol 289:416433.

Siegler MVS, Burrows M (1983) Spiking local interneurons as primary integrators of mechanosensory information in the locust. J Neurophysiol 50:1281-1295.

Siegler MVS, Burrows M (1986) Receptive fields of motor neurones underlying local tactile reflexes in the locust. J Neurosci 6:507-513.

Slifer EH (1954) The reaction of a grasshopper to an odorous material held near one of its feet (Orthoptera: Acrididae). Proc R Ent Soc Lond [A] 29:177-179.

Slifer EH (1956) The response of a grasshopper, Romalea microptera (Beauvois), to strong odours following amputation of the metathoracic leg at different levels. Proc R Ent Soc Lond [A] 31:95-98.

Stadler E, Hanson FE (1975) Olfactory capabilities of the "gustatory" chemoreceptors of the tobacco hornworm larvae. J Comp Physiol [A] 104:97-102.

White PR, Chapman RF (1990) Tarsal chemoreception in the polyphagous grasshopper Schistocerca americana: behavioural assays, sensilla distributions and electrophysiology. Physiol Entomol 15:105121. 